\title{
Characterization and Management of Borderline Ovarian Tumors - Results of a Retrospective, Single-center Study of Patients Treated at the Department of Gynecology and Obstetrics of the University Medicine Greifswald
}

\author{
DOMINIQUE KOENSGEN ${ }^{1 *}$, MARTIN WEISS ${ }^{2 *}$, KATHRIN ASSMANN $^{1}$, SARA Y. BRUCKER $^{2}$, \\ DIETHELM WALLWIENER ${ }^{2}$, MATTHIAS B. STOPE $^{3}$ and ALEXANDER MUSTEA ${ }^{1}$ \\ Departments of ${ }^{1}$ Gynecology and Obstetrics, and ${ }^{3}$ Urology, University of Medicine Greifswald, Greifswald, Germany; \\ ${ }^{2}$ Department of Gynecology and Obstetrics, University Hospital Tuebingen, Tuebingen, Germany
}

\begin{abstract}
Background/Aim: Borderline ovarian tumors (BOT) are malignant epithelial ovarian tumors with a very low incidence, therefore lacking sufficient clinical experience in diagnostics and treatment. This study characterized the histology, clinical features, diagnostics and therapy of BOT including patients treated at the Department of Gynecology and Obstetrics of the University Medicine Greifswald. Materials and Methods: In this retrospective, single-center study, patients with BOT treated between 1990 and 2010 were analyzed according to their histological and clinical reports. Results: A total of 54 patients were enrolled. The median age was 54.6 (range=23-83) years. Distribution of histological subtypes was: serous in 31 patients $(57.4 \%)$ and mucinous in 23 patients (42.6\%). All patients underwent surgery. Eight patients $(14.8 \%)$ were treated according to actual therapy recommendations during the initial surgery. Eight patients (14.8\%) received adjuvant chemotherapy contrary to treatment recommendations. In the case of 36 patients (66.7\%), a frozen section was taken intraoperatively, which matched the definitive histological result in $88.9 \%$. During average follow-up of 70.3 months (range=0-231 months), two patients (3.7\%) developed tumor recurrence after 9 and 29 months, respectively, two patients (3.7\%) died of causes other than BOT. Conclusion: Our study critically demonstrated that until a few years ago, BOTs were not usually treated
\end{abstract}

\footnotetext{
*These Authors contributed equally to this study.

Correspondence to: Matthias Stope, Department of Urology, University Medicine Greifswald, Ferdinand-Sauerbruch-Strasse, 17475 Greifswald, Germany. Tel: +49 38348680436, Fax: +49 38348680435, e-mail: matthias.stope@uni-greifswald.de
}

Key Words: Borderline ovarian tumor, BOT, treatment management. according to international therapy recommendations. Nevertheless, the rate of tumor recurrence was very low.

According to the International Federation of Gynecology and Obstetrics (FIGO) classification from 1971, borderline ovarian tumors (BOT) are tumors of "low malignant potential" but due to their epithelial origin - are considered malignant epithelial ovarian tumors (1-5). The latest classification of the World Health Organization (WHO) describes BOT as "atypical proliferative tumor" (6). The incidence of BOT ranges between 1.5 and 2.5 cases per 100,000 on the American continent $(1,7)$ and about 5 cases per 100,000 cases in Europe $(4,8)$. The mainly excellent prognosis of BOT of around $97 \%$ survival over 10 years is the result of the lack of destructive stromal invasion by the tumor cells $(9,10)$. Overall, $75 \%$ of BOTs present at FIGO stage I, meaning that the BOT is limited to the ovary. Among BOTs, six histological subtypes can be classified. Depending on the epithelial cell type, serous (50$55 \%)$, mucinous $(35-45 \%)$, endometrioid $(2-3 \%)$, clear cell $(<1 \%)$, seromucinous (5-7\%) and borderline Brenner tumor (3$5 \%$ ) can be distinguished (10). BOT can show microinvasion, and lymph node and peritoneal spread (11). These extraovarian manifestations of BOT critically influence the prognosis of the disease, however, there are still controversies about the optimal treatment regime. The criteria of diagnostics and treatment too often remain very broad, allowing certain scope for individual therapeutic decisions, especially when patients are very young and have not yet fulfilled their childbearing wish. Generally, there is consensus on the suitability of complete surgical resection including omentectomy, cytology following peritoneal lavage, peritoneal biopsies and in the case of mucinous BOT, appendectomy (12).

In this retrospective single-center study, we characterize the histology, clinical features, diagnostics and therapy of BOT among a collective of patients treated at the Department 
of Gynecology and Obstetrics of the University Medicine Greifswald between the years of 1990 and 2010.

\section{Patients and Methods}

Study design and data collection. This was a single-center retrospective study. Data were collected on 54 patients with the diagnosis of a BOT treated at Greifswald University of Medicine between 01.01.1990 and 31.12.2010. The patients were identified using the clinical and histopathological patient records preserved in the Institute's archive of the Department of Pathology and the Department of Gynecology and Obstetrics of University of Medicine Greifswald. Detailed information regarding symptoms, patient age, surgery, localization, adjuvant therapy and follow-up were obtained from clinical records. BOT diagnosis, histology and size were also extracted from pathology records. When clinical records were missing, the patient's personal physician was contacted in order to fill in a questionnaire. The study was performed in accordance with the principles of good clinical practice and the Declaration of Helsinki.

Statistical analysis. For statistical analysis, SPSS version 21 software (IBM Corp., Armonk, NY, USA) was used.

\section{Results}

Patient characteristics. A total of 54 patients treated due to a diagnosis of ovarian tumors of BOT between 01.01.1990 and 31.12.2010 at Greifswald University of Medicine were enrolled during this trial. The average age at time of diagnosis was 54.6 years (range $=23-83$ years). the majority of patients $(87 \%)$ underwent surgery at Greifswald University Hospital, whereas $5.6 \%$ of patients were treated at Wolgast Hospital, $3.7 \%$ of patients at Demmin Hospital and $1.9 \%$ of patients at Anklam and Ueckermünde hospitals, all being academic teaching departments of the Greifswald University of Medicine. Thirty-three patients $(61.1 \%)$ were postmenopausal and 16 patients $(29.6 \%)$ were still menstruating. Four patients (7.4\%) were post hysterectomy at the time of diagnosis and one patient $(1.9 \%)$ showed primary amenorrhea (Table I).

Body mass index. The body mass index was determined in 52 (96.3\%) cases using body weight and size. The average body mass index at the time of diagnosis was $28.1 \mathrm{~kg} / \mathrm{m}^{2}$ (range $\left.=18.4-52.0 \mathrm{~kg} / \mathrm{m}^{2}\right)$. Only one of the 54 women $(1.9 \%)$ was underweight, compared to $18(33.3 \%)$ with normal weight, $19(35.2 \%)$ who were moderately overweight, $12(22.2 \%)$ who were obese and two $(3.7 \%)$ who were morbidly obese.

Family history. Family history was ascertained for 49 patients $(90.7 \%)$. Twenty-eight of these patients $(51.9 \%)$ had tumor diseases in their family history. Among the 28 patients, firstdegree relatives were affected in 17 cases $(60.7 \%)$, and second-degree relatives were affected in the case of six patients $(21.4 \%)$. The relatives of 15 patients $(53.6 \%)$ had non-gynecological tumors such as cerebral tumors, melanoma, pancreatic and hematological cancer. The relatives of seven patients $(25.0 \%)$ had a history of breast cancer and in six cases $(21.4 \%)$, the relatives had a history of intestinal cancer.

General state of health and comorbidities. The general state of health and the presence of comorbidities was determined for 50 patients $(92.6 \%)$ using a verbal rating scale including 'good', 'moderately reduced' and 'reduced'. Forty patients (74.1\%) perceived their general state of health as 'good', whereas six patients $(11.1 \%)$ rated it as 'moderately reduced' and four $(7.4 \%)$ as 'reduced'.

Before surgery, tumors were symptomatic in 35 patients (64.8\%). Twenty-two patients $(40.7 \%)$ had upper/lower abdominal pain. Seven patients $(13.0 \%)$ each observed an increase of girth or alterations in body weight. Five patients $(9.3 \%)$ suffered from abnormities in their menstrual cycle, dysmenorrhea or climacteric afflictions. Other and less frequent symptoms were abdominal resistance, peritonism, dyspnea and pectanginous complaints.

Thirty-one patients $(57.5 \%)$ had cardiovascular diseases, $16(29.6 \%)$ had intra- or extra-genital diseases and 15 $(27.8 \%)$ had pulmonary diseases. Breast cancer was the most frequently diagnosed secondary tumor (six cases, $11.1 \%$ ).

Gynecological anamnesis. Overall, 53 out of the 54 patients (98.1\%) had had a menarche, whereas one patient $(2.9 \%)$ presented with primary amenorrhea. The average age at menarche was 13.5 years (range $=11-19$ years). In the case of six patients $(11 \%)$, the age at menarche was unknown. Thirty-three patients $(61.1 \%)$ were postmenopausal at time of diagnosis; the average age at menopause was 48.0 years (range $=33-58$ years). Patients enrolled had an average of 1.9 (range=0-6) pregnancies before the time of diagnosis. Of these, an average of 0.2 (range $=0-2$ ) were spontaneous abortions and an average of 0.3 (range, 0-2) were pregnancy interruptions. In addition, 10 patients $(18.5 \%)$ were never pregnant and one patient $(1.9 \%)$ had borne no viable child until the time of BOT diagnosis.

Smoking anamnesis. Fifteen out of the 54 patients $(27.8 \%)$ were cigarette smokers; 37 patients (68.5\%) were nonsmokers. Among smokers, 10 patients (18.5\%) consumed between 1 and 10 cigarettes per day, four (7.4\%) between 11 and 20 cigarettes per day and one $(1.9 \%)$ consumed more than 20 cigarettes per day.

Tumor markers. The assessment of CA125 (reference $=35 \mathrm{U} / \mathrm{ml})$ was performed in $41(75.9 \%)$ of the 54 patients and averaged $79.4 \mathrm{U} / \mathrm{ml}$ (range=4.6-1287.0 U/ml). In the case of 14 patients (34.1\%), CA125 exceeded the reference of $35 \mathrm{U} / \mathrm{ml}$. CA19-9 (reference $=37 \mathrm{U} / \mathrm{ml}$ ) was determined in 32 patients $(59.3 \%$ ). The average CA19-9 level was $1690.7 \mathrm{U} / \mathrm{ml}$ (range=0.3$34382.0 \mathrm{U} / \mathrm{ml})$. Ten patients $(18.5 \%)$ had values of CA19-9 above the reference level. 
Table I. Patient demographics.

\begin{tabular}{lc}
\hline No. of patients, $\mathrm{n}(\%)$ & $54(100)$ \\
Median age (range), years & $54.6(23-83)$ \\
Premenopausal, n (\%) & $16(29.6)$ \\
Postmenopausal, $\mathrm{n}(\%)$ & $33(61.1)$ \\
Primary amenorrhea & $1(1.9)$ \\
After hysterectomy & $4(7.4)$ \\
Body mass index $\left(\mathrm{kg} / \mathrm{m}^{2}\right)$ & \\
Median (range) & $27-0(18.4-52.0)$ \\
Underweight $(\%)$ & $1(1.9)$ \\
Nomal weight $(\%)$ & $18(33.3)$ \\
High weight $(\%)$ & 19 \\
Overweight $(\%)$ & $12(22.2)$ \\
Obesity (\%) & $2(3.7)$ \\
Unknown (\%) & $2(3.7)$ \\
Histology, $\mathrm{n}(\%)$ & \\
Serous & $31(57.4)$ \\
Unilateral & $23(42.6)$ \\
Bilateral & $8(14.8)$ \\
With microinvasion & $3(9.7)$ \\
With micropapillary structures & $24(77.4)$ \\
Mucinous & $23(42.6)$ \\
Unilateral & $20(37.0)$ \\
Bilateral & $2(3.7)$ \\
With intraepithelial carcinoma & $1(4.5)$ \\
\hline
\end{tabular}

Preoperative ultrasound. Fifty (92.6\%) out of 54 patients underwent ultrasound examination previous to surgery. Ultrasound was performed transvaginally in 28 patients $(51.9 \%)$, abdominally in 15 patients $(13.0 \%)$ and both transvaginally and abdominally in 15 patients $(27.8 \%)$. In all cases, a tumorous structure was detected in the ovaries.

Surgery. Each of the 54 patients (100.0\%) enrolled in this study underwent surgery at the time of diagnosis. Laparotomic incision was the most frequently chosen form of entry in 38 (70.4\%) out of the 54 patients. Laparoscopy was accomplished in eight patients (14.8\%). In the case of eight patients (14.8\%) undergoing laparoscopy, a conversion to laparotomy was indicated. Radicality of resection differed among the operations. In three patients (5.6\%), uni- or bilateral cysts were enucleated. Nine patients $(16.7 \%)$ underwent unilateral resection of the ovaries. Bilateral resection of the ovaries was the most abundant procedure in the case of 43 patients (79.6\%). Hysterectomy was the second most frequent procedure in 37 cases $(68.5 \%)$. Appendectomy was performed in 30 patients $(55.6 \%)$ and omentectomy was in 28 patients $(51.9 \%)$. Four patients $(7.4 \%)$ underwent omental biopsies. In 11 patients (20.4\%), peritoneal biopsies were sampled, whereas in four patients $(7.4 \%)$ a complete resection of the peritoneum was performed. A pelvic lymph node dissection was implemented in the case of nine patients (16.7\%), a resection of pelvic and para-aortic lymph nodes was performed in the case of five patients (9.3\%). Nineteen patients (35.2\%) underwent adhesiolysis, and ureterolysis was carried out in two patients $(3.7 \%)$. Moreover, 20 patients $(37.0 \%)$ underwent intraoperative peritoneal lavage. A frozen section analysis was performed for 36 patients (66.7\%). Intraoperative tumor rupture occurred in eight patients (14.8\%). Overall, in 53 patients $(98.1 \%)$ an $\mathrm{R} 0$ resection was obtained.

In summary, eight patients (14.8\%) underwent surgery according to the recent $\mathrm{S} 3$ guidelines for diagnostics, therapy and aftercare of malignant tumors of the ovaries published by the German Society for Gynecology and Obstetrics (Deutsche Gesellschaft für Gynäkologie und Geburtshilfe, DGGG) (13). Nine patients (16.7\%) underwent fertility-preserving surgery.

Adjuvant chemotherapy. Overall, eight patients (14.8\%) received adjuvant chemotherapy. Seven of these patients (87.5\%) had no evidence of residual disease after chemotherapy. In the case of one patient (1.9\%), the result of the adjuvant chemotherapy was unknown. Two patients (3.7\%) received six cycles of cyclophosphamide and carboplatin, whereas two (3.7\%) underwent four cycles and one patient $(1.9 \%)$ three cycles. One patient $(1.9 \%)$ received six cycles and another patient (1.9\%) four cycles of carboplatin. One patient (1.9\%) underwent six cycles of carboplatin and taxol.

Histology. Thirty-one (57.4\%) out of 54 samples were serous and $23(42.6 \%)$ were mucinous differentiated tumors. In three (9.7\%) out of the 31 serous tumors, microinvasion was detected. Twenty-four tumors (77.4\%) contained micropapillary structures. Moreover, one of the mucinous tumors $(4.5 \%)$ contained a carcinoma in situ. No case of pseudomyxoma peritonei was detected. Twenty-three of the serous $(42.6 \%)$ and 20 of the mucinous $(37.0 \%)$ tumors were located unilaterally, whereas eight of the serous (14.8\%) and two of the mucinous $(3.7 \%)$ tumors occurred bilaterally.

Definitive surgery and second-look operation. Eleven patients (20.4\%) underwent definitive surgery according to S3 treatment recommendations (13) after tumor diagnosis. Eight of these patients (14.8\%) underwent a laparotomy, two (3.7\%) a laparoscopy and one patient (1.9\%) underwent a laparoscopy-assisted vaginal hysterectomy. In five patients $(9.3 \%)$, the tumor was completely removed.

Twelve patients $(22.2 \%)$ underwent a second-look operation, most frequently by laparoscopy (nine patients, $16.7 \%)$. Thereby, tumor was found in two patients $(3.7 \%)$, and one of those $(1.9 \%)$ underwent definitive surgery following the second-look operation.

Post-surgical side-effects. Twenty-two patients (40.7\%) suffered from single or multiple post-surgical side-effects. Most frequently urological or renal disorders occurred in 10 patients 
(18.5\%), followed by wound-healing disorders and herniation in seven patients $(13.0 \%)$ and anemia in six (11.1\%). Four patients $(7.4 \%)$ reported gastrointestinal symptoms and two patients $(3.7 \%)$ had pulmonary complications. Furthermore, one patient (1.9\%) suffered from septic multi-organ failure and cardiac symptoms. One patient $(1.9 \%)$ died immediately after surgery. On average, hospitalization lasted 11.7 days ( median $=12$, range $=3-23$ days).

Aftercare. Aftercare took place through physicians in private practice or at the University Women's Hospital. Twelve patients $(22.2 \%)$ were re-examined yearly, another 12 patients $(22.2 \%)$ every 6 months, eight patients $(14.8 \%)$ were re-examined quarterly and two patients $(3.7 \%)$ participated in aftercare every 2-3 years. During aftercare, 33 patients $(61.1 \%)$ underwent gynecological examination, $21(38.9 \%)$ underwent transvaginal ultrasound and six (11.1\%) underwent transvaginal and abdominal ultrasound examination. Moreover, in the case of 20 patients $(37.0 \%)$, a general clinical examination was performed. CA125 level was determined in the case of eight patients $(14.8 \%)$.

Follow-up. The mean duration of follow-up was 70.3 months (median $=40.5$, range $=0-231$ months). Fourteen patients (25.9\%) died within the follow-up period, with a mean time to death of 225 months. There were no verified tumor-associated deaths. Two patients $(3.7 \%)$ showed a recurrence of disease. Overall, four patients $(7.4 \%)$ were lost to follow-up.

A 49-year-old patient showed tumor recurrence 9 months after primary laparotomy with bilateral adnexectomy, omentectomy, pelvic lymphadenectomy, abdominal lavage and excision of peritoneal sampling due to a $9 \mathrm{~cm}$ unilateral serous tumor with micropapillary structures. This patient had undergone a hysterectomy earlier. During primary surgery, tumor rupture was documented; the patient received no adjuvant therapy. After diagnosis of tumor recurrence, the patient underwent re-laparotomy and tumor debulking due to peritoneal carcinosis but died 11 months after primary diagnosis of the disease.

During a second-look operation another patient showed a tumor recurrence comprising a $10 \mathrm{~cm}$ low-grade mucininous carcinoma of the left ovary located at the liver and vaginal stump 29 months after the first diagnose.

\section{Discussion}

Various studies reported the occurrence of BOT as between 28 and 62 years and therefore, at an average of 10 years earlier compared to the ovarian cancer (14-21). Our data are comparable, with an average age of 54.6 (range=23-83) years. In line with our study, where $69 \%$ of 54 patients were found to be postmenopausal at the time of diagnosis, in previous investigations, $60 \%, 70 \%$ and $65 \%$ of women were postmenopausal at time of diagnosis $(18,22,23)$. In contrast, studies of Desfeux et al. (20), Ji et al. (24) and Pirimoglu et al. (25) revealed that only $37 \%, 41 \%$ and $28 \%$ of women with BOT were postmenopausal. In our collective, the average age of menopause was 48.0 years (range=33-38). Four patients $(7.4 \%)$ had undergone a hysterectomy when premenopausal. Furthermore, patients with BOT had an average age of menarche of 13.5 (range=11-19) years, which is comparable to several authors (13.0 to 14.6 years) $(15,19$, $20,22,26)$. Overall, no study showed any influence of the time point of menarche or menopause for the risk of subsequent BOT.

Riman et al. identified a risk-reductive effect of childbirth for both serous and mucinous BOT when comparing 193 patients to a control cohort (19). On average, patients with BOT had 1.8 births and 0.2 abortions. On average $17.1 \%$ of women were nulliparous. Other studies also showed that patients with BOT had fewer childbirths. as Ji et al. reported 1.9 (range $=0-8)$ pregnancies and 1.6 (range $=0-7)$ childbirths (20), and Ayhan et al. reported 2.1 (range=0-13) pregnancies and 1.9 (range=0-11) childbirths (22). In this study, $31 \%$ of patients with BOT were nulliparous. Moreover, Nikrui et al. (23) and Eltabbakh et al. (27) showed that $39 \%$ and $22 \%$ of patients with BOT were nulligravida. A meta-analysis of nine studies by Harris et al. (28) showed that parity was inversely proportional to the risk of developing BOT and that an increased number of childbirths was associated with a further risk reduction. Interestingly, infertile women had an increased risk for BOT development compared to controls without infertility in medical history. Concerning the number of pregnancies and childbirths, the patient collective in the present study did not substantially differ from those mentioned above and therefore our date strongly support the results of previous studies. Further research has been carried out in terms of risk reduction of BOT due to breast feeding and intake of oral contracepives. Studies by Harris et al. (28), Bernard et al. (29) and Riman et al. (19) showed that breast feeding reduces the risk of BOT development, whereas a study of Jordan et al. (30) detected no risk reduction. Very heterogenous results are associated with the intake of oral contraceptives. Harris et al. did not find any correlation between oral contraceptive use and the risk of BOT development (28), whereas Bernard et al., interestingly, detected a risk reduction (29) and Jordan et al. (30) showed a risk reduction of $50 \%$. In contrast, estrogen substitution for hormone replacement therapy increased the risk of BOT development in the study of Riman et al. (19).

This study aimed to determine a possible relation between tumor diseases in family history and the risk of developing a BOT. Surprisingly, 28 out of 54 patients (51.9\%) reported tumor events in their family history, $3.7 \%$ had events solely of breast cancer, whereas $9.3 \%$ had cases of breast cancer and another tumor type in their family; $1.9 \%$ of patients had 
solely colon carcinoma in their family history, $9.3 \%$ reported colon carcinoma and another tumor type. In a study by Nikrui et al., $13 \%$ of patients had a positive tumor anamnesis in their family (23). Riman et al. determined $8 \%$ of patients had cases of breast cancer in their family (19). Furthermore, Gotlieb et al. showed that $4.3 \%$ of their collective of 117 patients with BOT were positive for BRCA1/2 mutation compared to $24.2 \%$ among patients with invasive ovarian cancer (31). Overall, $8.2 \%$ of patients with BOT had cases of breast cancer in their family history. Various other studies did not identify a correlation between positive tumor family history and the risk of BOT development $(27,30,32)$.

Interestingly, this study found that $22.2 \%$ of the patients with BOT were diagnosed with other tumor diseases, among these the most frequent secondary tumor was breast cancer $(11.1 \%)$. Nikrui et al. also found that $16.2 \%$ of patients developed a secondary tumor when diagnosed with BOT (23). A study by Bouchardy et al. found that $12 \%$ of patients with BOT developed a secondary tumor, most frequently including breast cancer and colorectal cancer (14). The authors postulated a 3.97-fold increased risk for colorectal cancer development. The risk for breast cancer was assumed to be twice as high compared to patients without BOT.

In this study, $32.7 \%$ of patients with BOT were asymptomatic. When suffering from symptoms, pain of the upper and lower abdomen $(40.7 \%)$, alterations of body weight (7\%) and girth (7\%) were the most frequent events. These findings are in line with other studies, as Ji et al. found $69.5 \%$ of patients with BOT were symptomatic (20). Nikrui et al. (23) and Cusido et al. (33) found $14.0 \%$ and $30.4 \%$ of patients, respectively, were asymptomatic. However, abdominal pain and alterations of body weight and girth were the leading symptoms.

Beside the mostly unspecific symptoms of BOT, serum tumor markers such as CA125 and CA19-9 play an important role in BOT diagnostics. Among the patient collective in this study, the tumor markers CA125 and CA19-9 were preoperatively determined in $75.9 \%$ and $59.3 \%$ of cases, with an average of $79.4 \mathrm{U} / \mathrm{ml}$ and $1,690.7 \mathrm{U} / \mathrm{ml}$, respectively. CA125 was increased in $34.2 \%$, whereas CA19-9 was greater than the reference level in $31.3 \%$ of cases. Our results are in line with those of Wong et al. who found CA125 to be increased in $39 \%$ of his collective. Among 234 patients included in a study by Ren et al., CA125 and CA19-9 were increased in $62 \%$ and $11 \%$ of cases, respectively (17). CA125 thereby correlated with advanced-stage BOT, however, it was not an independent factor for the probability of tumor relapse. Pirimoglu et al. found an increased CA125 level in $57.8 \%$ (25), whereas Engelen et al. reported that $24 \%$ of patients with BOT had a CA125 level above the reference value and an increased CA19-9 level in 46\% (34). The authors suggested CA19-9 to be the more reliable tumor marker and recommended its determination during the follow-up of Bo's.
Overall, however, the determination of serum tumor markers seems to increase early detection of tumor recurrence in only a minority of cases.

Concerning the histological differentiation of BOT, there were no major differences between this study and the literature. Most recent studies report rates of between $55 \%$ and $66 \%$ for serous tumors, and $34 \%$ and $45 \%$ for mucinous tumors $(14,15,19,35,36)$, both being the most frequent histological differentiation of BOT. Some studies with other patient collectives found mucinous BOT to be more abundant (27, 34, 37). Ji et al. (20) and Donnez et al. (38) showed a nearly balanced distribution of serous (48\% and $49 \%)$ and mucinous ( $47 \%$ and $48 \%$ ) BOT. In this study, $25.8 \%$ of serous tumors and $8.7 \%$ of mucinous tumors were found to be bilateral. Except for the studies of Hung-Yaw et al. (27) and Ren et al. (17), this is in line with most of the recent research in this field $(20,22,34,35,37,39)$. Furthermore, serous tumors appeared to be greater in size compared to mucinous tumors $(20,27,36,40-42)$, which is validated by this study.

Concerning the actual German guidelines for diagnostics and therapy of malignant tumors of the ovary (13), the performance of a frozen section is not imperative. Among the collective of this study, an intraoperative frozen section was performed in $66.7 \%$ of patients. Of these, in $88.9 \%$, the diagnosis of the frozen section was concordant with the definitive histological result. This success rate is outstanding compared to the results of other studies in which it ranged between $60 \%$ and $78.4 \%(22,27,40,43,44)$. However, convincing studies on the definitive benefit of the performance of an intraoperative frozen section are still lacking.

Apart from this, the performance of peritoneal lavage and sample taking for cytology is recommended by international therapy guidelines and is part of adequate surgical staging (2, $7,13,45)$. In this study, peritoneal lavage followed by cytology was carried out in $72.2 \%$ of cases; among these, tumor cells were found in $7.4 \%$, and in $3.2 \%$, tumor cells could not be excluded. In spite of the fact that the performance of cytology is a simple and an effective method to diagnose peritoneal extension of BOT, only few studies describe the application of this technique $(15,46)$. In literature, the rate of cytology following peritoneal lavage was described as being between $21.1 \%$ and $72.2 \%(16,27)$. In total, $4.4 \%$ to $80.0 \%$ of these cytology samples tested positively for tumor cells.

There is no evidence that adjuvant chemo- or radiotherapy for BOT is associated with increased overall survival (13). Therefore, the only recommended therapy of BOT is radical surgery, including careful abdominal exploration, total hysterectomy, bilateral salpingo-oophorectomy, omentectomy, excision of all conspicuous lesions, performance of various peritoneal biopsies, and peritoneal lavage with subsequent cytology (12-13). According to actual therapy guidelines, conservative fertility-sparing surgery may be discussed with 
patients under 40 years of age and with incomplete childbearing $(12,13)$. After informed consent about the fourfold increased risk of tumor recurrence, this includes unilateral salpingooophorectomy or unilateral cystectomy, abdominal exploration, peritoneal lavage and cytology, omentectomy, excision of suspicious lesions and peritoneal biopsies $(2,13,47)$. In fact, in this study, only eight patients $(14.8 \%)$ primarily underwent surgical treatment according to therapy guidelines. Five more patients $(9.3 \%)$ completed the treatment according to therapy guidelines after a secondary surgery. Retrospectively, only one (1.9\%) out of nine $(16.7 \%)$ patients treated by fertility-sparing surgery had a serious wish for childbearing. Furthermore, eight patients $(14.8 \%)$ received adjuvant chemotherapy. The reasons why these treatment modes, divergent from actual therapy guidelines, were chosen between 1990 and 2010 is retrospectively difficult to comprehend.

During a mean follow-up of 70.3 months (median=40.5, range $=0-231$ months $), 14$ patients $(25.9 \%)$ died, with a mean time to death of 225 months. None of these deaths was tumor associated. In this study, two patients $(3.7 \%)$ experienced recurrence of disease 9 and 29 months after tumor resection. This is a very low percentage compared to literature, with stated rates of disease recurrence of up to $11.5 \%(16,33-35)$. Among these cases, tumor recurrence was observed within the first 10 years after initial surgery. Nevertheless, the mean duration of follow-up in literature was only 21 to 105.6 months $(14,16-18,20,24,33-35,37)$.

In conclusion, even though only eight out of 54 patients received surgical treatment of BOT according to actual therapy guidelines between 1990 and 2010. To date only, two cases of tumor recurrence were detected.

Interestingly, the number of patients who were primarily treated according to therapy guidelines has increased since 2007. However, further studies are necessary to investigate recent therapy procedures at the Greifswald University Hospital with its associated peripheral academic teaching departments Wolgast, Demmin, Anklam and Ueckermünde.

\section{References}

1 Morice P, Uzan C, Fauvet R, Gouy S, Duvillard P and Darai E: Borderline ovarian tumour: pathological diagnostic dilemma and risk factors for invasive or lethal recurrence. Lancet Oncol 13: e103-115, 2012.

2 Fischerova D, Zikan M, Dundr P and Cibula D: Diagnosis, treatment, and follow-up of borderline ovarian tumors. Oncologist 17: 1515-1533, 2012.

3 Yokoyama Y, Moriya T, Takano T, Shoji T, Takahashi O, Nakahara K, Yamada H, Yaegashi N, Okamura K, Izutsu T, Sugiyama T, Tanaka T, Kurachi H, Sato A, Tase T and Mizunuma $\mathrm{H}$ : Clinical outcome and risk factors for recurrence in borderline ovarian tumours. Br J Cancer 94: 1586-1591, 2006.

4 Trope CG, Kaern J and Davidson B: Borderline ovarian tumours. Best Pract Res Clin Obstet Gynaecol 26: 325-336, 2012.
5 International Federation of Gynecology and Obstetrics F: Classification and staging of malignant tumours in the female pelvis. Acta Obstet Gynecol Scand 50: 1-7, 1971.

6 Kurman RJ, Carcangiu ML, Herrington CS and Young RH: WHO Classification of Tumours of Female Reproductive Organs. Fourth Edition. Lyon, IARC, 2014.

7 Lalwani N, Shanbhoque AK, Vikram R, Nagar A, Jagirdar J and Prasad SR: AJR Am J Roentgenol 194: 330-336, 2010.

8 Ray-Coquard I, Pujade-Lauraine E, Pautier P, Méeus P, Morice P, Treilleux I, Duvillard P, Alexandre J, Vacher-Lavenu MC, Selle F, Guastalla JP and Lhommé C: Tumores infrecuentes del ovario: estrategias terapéuticas y organización del tratameinto. EMC Ginecol Obstet 48: 1-10, 2012.

9 Siverberg SG, Bell Da, Kurman RJ, Seidman JD, Prat J, Ronnett BM, Copeland L, Silva E, Gorstein F and Young RH: Borderline ovarian tumors: key points and workshop summary. Hum Pathol 35: 910-917, 2004.

10 Hauptmann S, Friedrich K, Redline R and Avril S: Ovarian borderline tumors in the 2014 WHO classification: evolving concepts and diagnostic criteria. Virchows Arch 470: 125-142, 2017.

11 Seidman JD and Kurman RJ: Ovarian serous borderline tumors: a critical review of the literature with emphasis on prognostic indicators. Hum Pathol 31: 539-557, 2000.

12 Abascal-Saiz A, Sotillo-Mallo L, de Santiago Jm and Zapardiel I: Management of borderline ovarian tumours: a comprehensive review of the literature. Ecancermedicalscience 8: 403, 2014.

13 Faluyi, O, Mackean M, Gourley C, Bryant A and Dickinson HO: Interventions for the treatment of borderline ovarian tumours, Cochrane Database Syst Rev 9: CD007696, 2010.

14 Bouchardy C, Fernandez S, Merglen A, Usel M, Fioretta G, Rapiti E, Schubert H, Pelte MF, Chappuis PO and Vlastos G: Increased risk of second cancer among patients with ovarian borderline tumors. Gynecol Oncol 109: 210-214, 2008.

15 Lenhard MS, Mitterer S, Kümper C, Stieber P, Mayr D, Ditsch $\mathrm{N}$, Friese $\mathrm{K}$ and Burges A: Long-term follow-up after ovarian borderline tumor: Relapse and survival in a large patient cohort. Eur J Obstet Gynecol 145: 189-194, 2009.

16 Camatte S, Morice P, Thoury A, Fourchotte V, Pautier P, Lhomme $\mathrm{C}$, Duvillard $\mathrm{P}$ and Castaigne D: Impact of surgical staging in patients with macroscopic "stage I" ovarian borderline tumours: analysis of a continuous series of 101 cases. Eur J Cancer 40: 1842-1849, 2004.

17 Ren J, Peng Z and Yang K: A clinicopathologic multivariate analysis affecting recurrence of borderline ovarian tumors. Gynecol Oncol 110: 162-167, 2008.

18 Boran N, Cil AP, Tulunay G, Ozturkoglu E, Koc S, Bulbul D and Kose MF: Fertility and recurrence results of conservative surgery for borderline ovarian tumors. Gynecol Oncol 97: 845851, 2005.

19 Riman T, Dickmann, PW, Nilsson S, Correia N, Nordlinder H, Magnusson, CM and Persson IR: Risk factors for epithelial borderline ovarian tumors: results of a Swedish case-control study. Gynecol Oncol 83: 575-585, 2001.

20 Ji H, Yliskoski M, Anttila M, Syrjänen K and Saarikoski S: Management of stage-I borderline ovarian tumors. Int J Gynecol Obstet 54: 37-44, 1996.

21 Segal GH and Hart WR: Ovarian serous tumors of low malignant potential (serous borderline tumors), the relationship of exophytic surface tumor to peritoneal "implants". Am J Surg Pathol 16: 577-583, 1992 
22 Ayhan A, Guvendag Guven ES, Guven S and Kucukali T: Recurrence and prognostic factors in borderline ovarian tumors. Gynecol Oncol 98: 439-445, 2005.

23 Nikrui N: Survey of clinical behavior of patients with borderline epithelial tumors of the ovary. Gynecol Oncol 12: 107-119, 1981.

24 Desfeux P, Camatte S, Chatellier G, Blanc B, Querleu D and Lecuru F: Impact of surgical approach on the management of macroscopic early ovarian borderline tumors. Gynecol Oncol 98: 390-395, 2005.

25 Pirimoglu ZM, Afsin Y, Guzelmeric K, Yilmaz M, Unal O and Turan MC: Is it necessary to do retroperitoneal evaluation in borderline epithelial ovarian tumors? Arch Gynecol Obstet 277: 411-414, 2008.

26 Chang HY, Lin H, Changchien CC and Chang SY: Management of ovarian borderline malignancy. Taiwanese J Obstet Gynecol 43: 16-19, 2004.

27 Eltabbakh GH, Natarajan N, Piver MS and Mettlin CJ: Epidemiologic differences between women with borderline ovarian tumors and women with epithelial ovarian cancer. Gynecol Oncol 74: 103-107, 1999.

28 Harris R, Whittemore AS, Itnyre J, and the Collaboratve Ovarian Cancer Group: Characteristics relating to ovarian cancer risk: collaborative analysis of 12 US case-control studies, iii. epithelial tumors of low malignant potential in White women. Am J Epidemiol 136: 1204-1211, 1992.

29 Harlow BL, Weiss NS, Roth GJ, Chu J and Daling JR: Casecontrol study of borderline ovarian tumors: reproduktive history and exposure to exogenous female hormones. Cancer Res 48 : 5849-5852, 1988

30 Jordan SJ, Green AC, Whiteman DC and Webb PM for the Australian Cancer Study (Ovarian Cancer) and the Australian Ovarian Cancer Study Group: Risk factors for benign, borderline and invasive mucinous ovarian tumors: Epidemiological evidence of a neoplastic continuum? Gynecol Oncol 107: 223-230, 2007.

31 Gotlieb WH, Chetrit A, Menczer J, Hirsh-Yechezkel G, Lubin F, Friedman E, Modan B and Ben-Baruch G for the National Israel Ovarian Cancer Study Group: Demographic and genetic characteristics of patients with borderline ovarian tumors as compared to early-stage invasive ovarian cancer. Gynecol Oncol 97: 780-783, 2005.

32 Auranen A, Grenman S, Mäkinen J, Pukkala E, Sankila R and Salmi T: Borderline Ovarian Tumors in Finnland: Epidemiology and Familial Occurrence. Am J Epidemiol 144: 548-553, 1996.

33 Cusido M, Balaguero L, Hernandez G, Falcon O, RodriguezEscudero FJ, Vidart JA, Zamora L, Monera M and Alonso A, Section of Gynecologic Oncology and Breast Pathology of the Spanish Fedaration of Gynecologic Oncology (SEGO): Results of the national survey of borderline ovarian tumors in Spain. Gynecol Oncol 104: 617-622, 2007.

34 Engelen MA, de Bruijn HW, Hollema H, ten Hoor KA, Willemse PH, Aalders JG and van der Zee AG: Serum CA 125, Carcinoembryonic antigen, and CA19-9 as tumor markers in borderline ovarian tumors. Gynecol Oncol 78: 16-20, 2000.
35 Romagnolo C, Gadducci A, Sartori E, Zola P and Maggino T: Management of borderline ovarian tumors: Results of an Italian multicenter study. Gynecol Oncol 101: 255-260, 2006.

36 Rice LW, Lage J, Berkowitz RS, Goodman A, Muto MG, Knapp $\mathrm{RC}$ and Bell DA: Preoperative Serum CA-125 levels in borderline tumors of the ovary. Gynecol Oncol 46: 226-229, 1992.

37 Wong HF, Low JJ, Chua Y, Busmanis I, Tay EH and Ho TH: Ovarian tumors of borderline malignancy: a review of 247 patients from 1991 to 2004, 2007 IGCS and ESGO. Int J Gynecol Cancer 17: 342-349, 2007.

38 Donnez J, Munschke A, Berliere M, Pirard C, Jadoul P, Smets M and Squifflet J: Safety of conservative management and fertility outcome in women with borderline tumors of the ovary. Fertil Steril 79: 1216-1221, 2003.

39 Wu TI, Lee CL, Wu MY, Hsueh S, Huang KG, Yeh CJ and Lai $\mathrm{CH}$ : Prognostic factors predicting recurrence in borderline ovarian tumors. Gynecol Oncol 114: 237-241, 2009.

40 Houck K, Nikrui N, Duska L, Chang Y, Fuller AF, Bell D and Goodman A: Borderline Tumors of the ovary: correlation of frozen and permanent histopathologic diagnosis. Obstet Gynecol 95: 839-843, 2000.

41 Tempfer CB, Polterauer S, Bentz EK, Reinthaller A and Hefler LA: Accuracy of intraoperative frozen section analysis in borderline tumors of the ovary: A retrospective analysis of 96 cases and review of the literature. Gynecol Oncol 107: 248-252, 2007.

42 Neunteufel W, Gitsch G, Schieder K, Kölbl H and Breitenecker G: Karzinome niedriger maligner Potenz (Borderline-Tumore) des Ovars: Immunmorphologie und Klinik. Arch Gynecol Obstet 245: 600-602, 1989.

43 Fauvet R, Poncelet C, Boccara J, Descamps P, Fondrinier E and Darai E: Fertility after conservative treatment for borderline ovarian tumors: a French multicenter study. Fertil Steril 83: 284290, 2005.

44 Fauvet R, Boccara J, Dufournet C, Poncelet C and Darai E: Laparoscopic management of borderline ovarian tumors: results of a French multicenter study. Ann Oncol 16: 403-410. 2005.

45 Morice P, Uzan C and Gouy S: Cirugia de los tumores epiteliales malignos del ovario. EMC-Ginecol Obstet 10: 1-16, 2010.

46 Lackman F, Carey MS, Kirk ME, McLachlin CM and Elit L: Surgery as sole treatment for serous borderline tumors of the ovary with noninvasive implants. Gynecol Oncol 90: 407-412, 2003.

47 Park JY, Kim DY, Kim JH, Kim YM, Kim YT and Nam JH: Surgical management of borderline ovarian tumors: The role of fertility-sparing surgery. Gynecol Oncol 113: 75-82, 2009.

Received September 12, 2017

Revised October 17, 2017

Accepted October 18, 2017 